\title{
Satisfacción de la imagen corporal en universitarios que cuenten con el 10\% del cuerpo tatuado y universitarios no tatuados 4
}

\author{
Julián Esteban Ballén Valderrama \\ Psicólogo \\ Corporación Universitaria Iberoamericana \\ Bogotá, Colombia \\ Correo: julianballenpsyque@hotmail.com
}

\author{
Fernando Riveros Munévar \\ Magister em Psicología \\ Corporación Universitaria Iberoamericana \\ Bogotá, Colombia \\ Correo: efriveros45@hotmail.com
}

Recibido: $12 / 05 / 2015$

Evaluado: 22/07/2015

Aceptado: 22/09/ 2015

Objetivo: identificar la satisfacción de la imagen corporal en universitarios con mínimo el 10\% de sus cuerpos tatuados y universitarios sin ningún tipo de modificación corporal. Método: estudio no experimental de diseño transaccional descriptivo, se realizó con una muestra de 34 universitarios tatuados (11 mujeres - 23 hombres) y 82 universitarios sin modificaciones corporales (41 mujeres y 41 hombres) mayores de 20 años, residentes en Bogotá, vinculados a un programa de educación superior, sin ningún antecedente psiquiátrico y que no pertenecieran a ninguna tribu urbana 0 grupo social específico. Resultados: se evidenciaron diferencias significativas frente a la satisfacción general de la imagen corporal siendo los universitarios tatuados quienes presentan mayores puntajes. De igual manera, se halló que tanto las mujeres como los hombres con el 10\% del cuerpo tatuado presentan diferencias significativas favorables de imagen corporal en relación a aquellas personas que no se han modificado. Conclusiones: se atribuye al tatuaje funcionalidad para el reconocimiento mental del cuerpo y la autoevaluación permitiendo tener un juicio valorativo más satisfactorio en el individuo sobre su imagen corporal.

Palabras clave Satisfacción de la imagen corporal, Tatuaje, Modificación Corporal, estudiantes universitarios.

4 Para citar este artículo: Ballén, A., \& Riveros, F. (2015). Satisfacción de la imagen corporal en universitarios que cuenten con el 10\% del cuerpo tatuado y universitarios no tatuados. Informes Psicológicos, 15(2), 67-92. http:// dx.doi.org/10.18566/infpsicv15n2a04 


\section{Satisfaction of body image in college students with $10 \%$ of their bodies tattooed and those who have not tattooed their body}

Abstract

Aim: To identify the body image satisfaction in college students with at least $10 \%$ of their bodies tattooed and college students without any kind of body modification. Method: Non experimental study with a transactional descriptive design; it was conducted with a sample of 34 tattooed college students (11 women - 23 men) and 82 students without body modifications (41 women and 41 men) older than 20 years of age, residents of Bogotá, linked to a program of higher education, without any previous psychiatric history and who do not belong to any urban tribe or specific social group. Results: Significant differences on the general body image satisfaction were found, where tattooed students have higher scores. Similarly, it was found that both women and men with $10 \%$ of their body tattooed present significant favorable differences of body image in contrast with those persons who have not been modified. Conclusions: The tattoo is attributed with functionality for the mental recognition of the body and one's self-assessment allowing a more satisfactory evaluative judgement on the individual about their body image.

Keywords body image satisfaction, tattoo, body modification, college students.

\section{Satisfação da imagem corporal em universitários que contém com 10\% do corpo tatuado e universitários não tatuados}

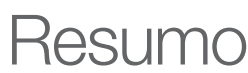

Objetivo: Identificar a satisfação da imagem corporal em universitários com mínimo de $10 \%$ de seus corpos tatuados e universitários sem nenhum tipo de modificação corporal. Método: Estudo não experimental de desenho transacional descritivo, se realizou com uma amostra de 34 universitários tatuados (11 mulheres - 23 homens) e 82 universitários sem modificações corporais (41 mulheres e 41 homens) maiores de 20 anos, residentes em Bogotá, vinculados a um programa de educação superior, sem nenhum antecedente psiquiátrico e que não pertenceram a nenhuma tribo urbana ou grupo social específico. Resultados: Se evidenciaram diferencias significativas frente à satisfação geral da imagem corporal sendo os universitários tatuados quem apresentam maiores pontuações. De igual maneira, se encontrou que tanto as mulheres como os homens com $10 \%$ do corpo tatuado apresentam diferencias significativas favoráveis de imagem corporal em relação a aquelas pessoas que não se hão modificado. Conclusões: Se atribui à tatuagem funcionalidade para o reconhecimento mental do corpo e a auto-avaliação permitindo ter um juízo valorativo mais satisfatório no individuo sobre sua imagem corporal.

satisfação da imagem corporal, tatuagem, modificação corporal, estudantes universitários. 


\section{ntroducción}

Los seres humanos cuentan por naturaleza con un cuerpo; este es visto como la realidad bio-fisiológica que permite la relación con el ambiente (Hurtado, 2008) y es definido como el componente más externo del ser humano el cual posibilita ejecutar acciones (Pérez \& Sánchez, 2001). Además de esta realidad física se encuentra un cuerpo que se origina socialmente cuando se tienen juicios y criterios aprendidos a través de la educación, humanización y socialización del individuo (Salinas, 1994).

Uno de los conceptos que involucra la representación corporal es considerado uno de los fenómenos más abordados en la actualidad denominado Imagen Corporal (Atencio, Molina \& Rojas, 2008). Es importante señalar que los primeros autores en describir este concepto lo definieron como la representación del cuerpo, el cual es construido en la mente, es decir, cómo parece y que juicio se tiene del cuerpo (Schilder, Krapf, Loedel, \& Corsico, 1983). Luego de esto se determinó que la imagen corporal está socialmente determinada; desde que se nace existen las influencias sociales que acentúan la autopercepción del cuerpo influyendo en el procesamiento de información puesto que gestiona la percepción del mundo a través de la forma en que un individuo siente y piensa de su cuerpo (Cash \& Pruzinsky, 1990). Más adelante se plantea que el término de imagen corporal mantiene unos componentes perceptivos y cognitivo-emocionales, pero además de ellos incluye un componente conductual que consistiría en qué conductas realiza el individuo en relación a su cuerpo y el grado de satisfacción con él, por ejemplo cuando se evitan situaciones donde el cuerpo se vea desnudo (Thompson, 1990, citado por Cucarella, 2013). En la actualidad la imagen corporal se define cómo la unión de sentimientos y actitudes alojadas en la memoria que son evocadas al percibir el cuerpo (Gallego, 2009) y para poder construir esta representación mental, el individuo necesita de referentes sociales, biológicos, psicológicos y económicos los cuales llegan a originar patrones estético corporales para cada individuo (Chaves, 2004, citado por Moreno \& Ortiz, 2009).

Ahora bien, cuando un sujeto que evalúa sus dimensiones corporales manifiesta juicios valorativos que no coinciden con las dimensiones reales presenta una alteración de la imagen corporal (Sepúlveda, Gandarillas, \& Carrobles, 2004). Las primeras investigaciones de este fenómeno proponen que la distorsión de la imagen corporal se evidencia a través de la sobre-estimación del tamaño (Bruch, 1962). Luego de esto se añade la existencia de sub-estimación del tamaño del cuerpo como complemento a los anteriores postulados (Garner \& Garfinkel, 1981), hasta que investigaciones más avanzadas proponen que estos dos conceptos son producto de una distorsión perspectiva de la talla combinada con la alteración cognitivo-afectivo de la preocupación y angustia que como consecuencia suscita un gran nivel de incapacidad e inestabilidad en términos emocionales (Espina, Ortego, Alda, Yenes, \& Alemán, 2001). Más adelante se determinaría que las alteraciones de la imagen corporal serían el resultado de una perturbación en el funcionamiento cortical que puede generar síndrome del miembro fantasma, prosopagnosia, 
anorexia entre otras patologías (Raich, 2004). De igual manera se postula que la Imagen Corporal es una preocupación exagerada, que produce malestar, hacia algún defecto imaginario o extremado de la apariencia física denominado Dismorfofobia Corporal (Argüello \& Romero, 2012). En términos conductuales, se evidencia que la insatisfacción de la imagen corporal se evidencia en consumir gran cantidad de horas al día comprobando el aspecto ante un espejo, pueden haber comportamientos de limpieza y aseo excesivo, algunos evitan mirarse al espejo o lo hacen indiscriminadamente, solicitan constantemente aprobación o información de sus defectos, realizan maniobras de camuflaje o suelen taparse con vestimentas holgadas que disimulen su insatisfacción (Raich, 2004).

Según Fernández, Otero, Castro y Prieto (2003) las mujeres a diferencia de los hombres muestran mayor preocupación por el peso y la imagen corporal, siendo ellas quienes en mayor medida recurren a dietas para alcanzar su peso ideal y manifiestan mayor número de variables cognoscitivas y comportamentales para adquirir trastornos de la conducta alimentaria. Otras investigaciones respaldan el postulado anterior pero en término de ansiedad y estrés; Vargas (2011) encontró que mujeres adolescentes experimentan mayor ansiedad en lo que se refiere a las áreas corporales que tienen que ver con el factor peso, muslos, nalgas, caderas, abdomen, piernas y cintura. Por otro lado se han encontrado diferencias significativas de insatisfacción de la imagen corporal entre mujeres con un trastorno de la conducta alimentaria y mujeres sin ningún antecedente de este tipo, por lo cual es necesario un manejo terapéutico pertinente que evite mayores dificultades en esta población (Espina et al, 2001). Otras investigaciones demuestran que mujeres sin trastornos de conducta alimentaria pero de mayor edad a la adolescencia presentan preocupación por el ideal de delgadez y la insatisfacción corporal (Álvarez, Franco, López, Mancilla, \& Vázquez, 2009). Así mismo diferentes investigaciones hallaron en mujeres que un miedo a ganar peso condiciona un menor nivel de experiencia sexual, también se halló que a mayor insatisfacción corporal menor experiencia sexual lo que permite pensar que la preocupación por este aspecto influye en las relaciones íntimas del sexo femenino (Otero, Fernández, \& Castro, 2004).

Ahora bien, Ibarzabal y Tubio (2008) hallaron que los hombres fisiculturistas competidores y no competidores suelen presentar aspectos comportamentales reflejo de la satisfacción o insatisfacción de la imagen corporal; el grupo de fisiculturistas competidores suelen compararse constantemente con sus compañeros de gimnasio, evitar mostrar su cuerpo fuera de un entorno deportivo, mirarse constantemente al espejo y pesarse; no se hallan diferencias entre los grupos frente a la dinámica de preocuparse por su imagen corporal. Por otro lado un estudio realizado en México muestra que uno de cada diez hombres que asisten al gimnasio evidencia preocupación grave por su imagen corporal por lo que se presentan rasgos relacionados a Vigorexia (Ayensa, Martínez, \& Rancel, 2005).

A partir de los resultados anteriores se entiende que el sexo femenino es quien más índice de insatisfacción corporal evidencia en las investigaciones y de igual manera quienes más métodos invasivos 
como la cirugía estética utiliza para remediar su dificultad.

\section{Modificaciones corporales}

La imagen corporal ha sido abordada de acuerdo a los cambios que se pueden realizar en ella a través de la cirugía estética, fenómeno que se ha incrementado notablemente durante los últimos años puesto que parte de la población cada vez es más infeliz con su aspectos corporal (Aafjes, 2008). Décadas atrás se logró determinar que parte de las personas que se someten a la modificación corporal a través de la cirugía plástica pueden estar estrechamente unidos con la enfermedad mental, más específicamente con ansiedad, depresión y baja autoestima (Jacobson, Edgerton, Meyer, Canter, \& Slaughter, 1960). Además se determinó que esa insatisfacción corporal es manifestada en mayor número por mujeres quienes evidencian problemas con su apariencia desde la década de 1800 (Castle, Honigman, \& Phillips, 2002). Este problema con la imagen corporal ha trascendido en el tiempo permitiendo demostrar las consecuencias negativas que tiene para un individuo, en la actualidad se ha demostrado bajo autoestima, problemas interpersonales de ansiedad dificultando la interacción social, problemas en las relaciones sexuales, problemas depresivos y desórdenes de la alimentación que cómo consecuencia pueden llevar a un procedimiento de cirugía plástica. (Montaño, 2006; Tausk \& Nousari, 2001).

De igual forma se ha determinado que muchos de los efectos de una cirugía estética pueden ser negativos, generando una mayor problemática al paciente (Sarwer, Pertschuk, Wadden \& Whitaker, 1998), entre estas se halla rencor hacía el cirujano, aislamiento social, conductas autodestructivas, disfunción familiar y depresión (Bolton, Pruzinsky, Cash, \& Persing, 2003). Para determinar lo anterior se han diseñado investigaciones con el fin de generar instrumentos que midan la satisfacción del paciente luego de una cirugía como es el caso del Body-Qol, herramienta para determinar el agrado de una cirugía de contorno corporal (Danilla et al. 2013).

Algunos estudios sugieren que existen pacientes que se sienten satisfechos con el resultado de una operación estética y se refleja en términos de mayor autoestima, disminución de la angustia, timidez y mayor calidad de vida (Castle, Honigman, \& Phillips, 2002). Por otro lado, algunas investigaciones realizadas determinan que existe una mayor satisfacción con la parte del cuerpo que se ha modificado a través de la cirugía y así mismo un incremento subyacente de autoconfianza (Crerand, Cash, \& Whitaker, 2006), pero en contraposición Vlazny (2002, citado por Rasines, 2012) expone que no se puede obtener un beneficio en la autoestima y autoconcepto de las personas a través de una cirugía debido a que, la autoestima es una cuestión de identidad básica, esencial para la validación personal y para la capacidad de experimentar la alegría; una vez conseguido, se trata de adentro hacia afuera y no en todos los casos de puede considerar que el crecimiento es de afuera hacia adentro.

Además de la cirugía plástica se encuentra una gran cantidad de formas para realizar modificaciones corporales, entre estas existe las Escarificaciones que 
son marcas o "estigmas" dejados en la piel mediante heridas, a través de cortes con distintos objetos punzantes (cuchillos, bisturís, etc.), con las cuales se forma un diseño o dibujo, también está el piercing que consiste en insertar en diversas zonas del cuerpo pequeñas joyas o aros los cuales deben ser de materiales específicos como acero quirúrgico, titanio, niobio, teflón, para así evitar el rechazo o infección de la zona decorada (López, 2007). Además de las anteriores existen otras técnicas más elaboradas como el branding, un tipo de escarificación que se basa en realizar una marca por medio de láser o con hierro candente y el microdermal que es una técnica que radica en el implante de piezas de titanio por debajo de la piel moldeándola de acuerdo a las necesidades estéticas del paciente (Manca, 2011).

\section{Tatuaje}

Una de las modificaciones corporales más populares en la actualidad es el Tatuaje, este consiste en realizar marcas o dibujos permanentes mediante la inyección de pigmentos y tintes especializados en la capa intermedia de la piel (Dermis) la cual no renueva sus células garantizando que la tinta permanezca allí; para esto se utiliza una maquina creada por el artista e inventor estadounidense Samuel O'Reillya fines del 1800 y que a través de una aguja esterilizada penetraen la piel a una velocidad de 50 y 3000 veces por minuto, con lo cual se va plasmando un diseño en la piel (Álvarez, 2000). Esta última es una de las técnicas de modificación corporal más antigua que existe, se encuentran registros de hace 5300 años, prueba de esto son los restos momificados de un cazador del neolítico conocido con el nombre de "Oetzi", encontrado en un glaciar de los Alpes el cual expone rodillas y espalda tatuadas (Torres, 2007). Por otro lado se encontraron los restos de la sacerdotisa de origen egipcio Amounet, quien se creé vivió en el año 2000 A.C. y muestra su cuerpo totalmente cubierto por dibujos decorativos relacionados a un componente sagrado y religioso ( $\mathrm{Te}$ sone, 2000). Cerca del año 1000 A.C. se conoce la práctica oriental de tatuarse y fue implementado como técnica de indumentaria y similitud del kimono (Ganter, 2005). En china comenzó a ser fuente de información no verbal que dependiendo del color, la cantidad y la región donde fuese ubicado representaba rasgos de belleza, estado civil y actividades específicas (Cassab, 2002). Por su parte los griegos y romanos utilizaban el tatuaje para señalar el rango y posición social, además funcionaba para diferenciar jerarquías militares y propiedad de un esclavo (Hermosillo, Tovar, Gómez, Herrera, \& Sánchez, 2011). Fue con la llegada de un marinero del océano pacifico conocido como el capitán Cook cerca del siglo XVIII que se comenzó a dispersar la práctica de tatuarse en la cultura occidental, esto lo hizo después de uno de sus viajes por el pacífico cuando descubrió la cultura Polinesia, donde uno de los comportamientos culturales era golpear con un hueso acanalado las pieles de los integrantes de la tribu para así generar una marca imborrable (Rodríguez et. al, 2012).

A medida que el ser humano modificó su lenguaje, pensamiento y comportamiento, el tatuaje se incorporó en nuevos territorios y a su vez con nuevas funcionalidades como sucedió en los años 60 y 70 con los hippies, quienes los adoptaron como símbolo de rebeldía, abandonando 
los motivos marineros y realizándose grandes diseños muy coloridos, acordes con la época (Torres, 2007). A su vez subculturas de la época como los teddy boy, bikers o los Hell's Angels en la década de los 60' y los punk's y skin's en los 70 usaron el tatuaje como símbolo de desobediencia hacia las reglas en la sociedad, manteniendo así el deseo por comunicar información y creencias de manera no verbal (Pierrat, Guillon, Joly, \& Lesven, 2000). Luego de esto se comenzó con la era de la "profesionalización del tatuaje" donde se tecnificaron y especializaron los procedimientos para realizarse un tatuaje provocando una masificación del mismo y logrando que en la década de 1980 se realizara la apertura de locales de tatuajes con las reglas sanitarias y utensilios convenientes, convirtiendo la práctica como un negocio que satisface necesidades económicas y estéticas (Le Breton, 2002).

El tatuaje ha sido abordado desde investigaciones de tipo cualitativo, Manca (2011); Sastre (2011) junto con Soto y Morett, (2004) plantean que al realizarse un tatuaje se genera un acto de diferenciación y singularidad, un sentimiento de propiedad hacía el cuerpo que provee beneficios en términos de identidad y socialización debido a que permite la integración del adolescente a un grupo que genera reconocimiento y admiración a corto plazo. Otras investigaciones postulan que la principal motivación de un tatuaje es perpetuar la presencia del mundo interno, los pensamientos y emociones que acompañan al ser humano, de acuerdo con esto el tatuaje implica una perspectiva de subjetividad humana que diferencia las características y experiencias de un individuo a otro (Pérez, 2009; Yépez \& Arzápalo, 2007).Por otro lado investigaciones psicodinámicas proponen que el tatuaje se relaciona a la tendencia humana por emerger y ser reconocido, por lo tanto se considera esta práctica como un factor determinante de narcisismo, un amor propio que gira en búsqueda de tener rasgos únicos que diferencien la persona y la hagan exclusiva frente a la sociedad (Sastre, 2011).

En cuanto a investigaciones de tipo Cuantitativo se encuentran muy pocas evidencias relacionadas con el tatuaje, algunas investigaciones proponen que en la actualidad tanto hombres como mujeres realizan la práctica de tatuarse, las mujeres prefieren hacerlo en la espalda, vientre, cuello y cadera, zonas que están relacionadas con el erotismo femenino, en cambio los hombres prefieren los brazos, espalda, pecho y canilla que se relaciona con la fuerza y virilidad (Pérez \& Sánchez, 2001). Estudios en EEUU y Argentina señalan factores de riesgo asociados a la presencia de los tatuajes en la adolescencia, por ejemplo en varones se ha determinado una relación entre tatuajes y conductas violentas, peleas, drogas pesadas, promiscuidad; en mujeres los tatuajes se relacionan con drogas pesadas, conductas sexuales y suicidios, además, estas investigaciones postulan que existen mayores probabilidades de un ambiente familiar disfuncional, monoparental, bajos ingresos económicos y baja escolaridad de los padres en adolescentes tatuados; también se encuentra que entre las drogas más reconocidas está el tabaco, alcohol y marihuana; respecto al área académica existen mayores probabilidades de fracaso escolar, ausencias sin permiso en repetidas ocasiones y además existe una tendencia de iniciar la actividad sexual a menor edad traducida a promiscuidad; para terminar gran 
porcentaje de adolescentes reconocen el tatuaje como una marca que será parte de su piel toda la vida pero ninguno muestra un grado de arrepentimiento de ello (Bosello et al, 2009; Busaniche, Eynamm, Mulli, Paz \& Catsicaris 2006; Carroll, Riffenburgh, Roberts, \& Myhre 2002; Pozgain, Barkic, Filakovic, \& Koic 2004; Roberts \& Ryan, 2002; Stephens, 2003).

Siendo así se clasifica el tatuaje como un método de modificación corporal que una vez es plasmado en el cuerpo puede generar cambios a nivel autopercepción de un individuo, por tal razón es necesario identificar si ese tatuaje genera cambios positivos o negativos frente a la imagen corporal en el ser humano permitiendo identificar si es un comportamiento funcional o disfuncional para el desarrollo integral del mismo.
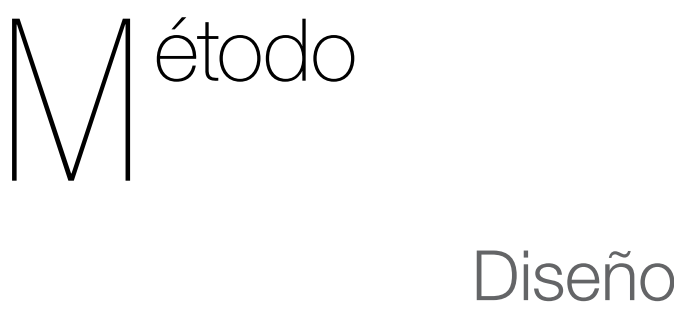

El presente es un estudio no experimental de tipo cuantitativo y de alcance descriptivo (Hernández, Fernández, \& Baptista, 2010).

\section{Participantes}

34 universitarios que cuenten con mínimo el diez por ciento (10\%) del cuerpo tatuado sin importar la ubicación del tatuaje y 82 estudiantes universitarios sin ningún tipo de modificación corporal.
Los integrantes de la población tienen mínimo veinte años, se encuentran cursando algún programa de educación superior, no cuentan con antecedentes psiquiátricos o psicológicos que evidencien diversidad cognitiva y tampoco han sido diagnosticados con algún trastorno mental o enfermedad médica que involucre disfuncionalidad mental. No hicieron parte de la muestra personas que hayan recibido medicación de por vida que manifieste efectos colaterales hacia el comportamiento, percepción o cognición, y tampoco fueron parte de la población aquellas personas que pertenecieran a alguna tribu urbana o grupo social que pueda ejercer influencia en su comportamiento. Por otro lado los participantes no podían haber consumido ningún tipo de sustancia psicoactiva con veinticuatro (24) horas de anterioridad, tampoco ningún tipo de medicamento que genere cambios perceptuales o comportamentales y en caso de que hayan tenido dolores físicos intensos o dificultades para conciliar el sueño se evaluará la decisión de administrar la prueba.

Con este tamaño muestral, la posibilidad de error es de 418906 (Faul, 2008), lo cual está por debajo del error esperado, permitiendo la validez de los datos y la posibilidad de generalizar los hallazgos a obtener.

\section{Instrumentos}

En primera medida se usará el esquema denominado "Regla de Nueve" descrito inicialmente por Pulaski y Tennison (1947, citado por Hoyos, Jaramillo, Molina, Valverde, \& Posso, 2007); este es un 
esquema corporal humano en el cual se asigna un porcentaje a cada área del cuerpo, de esta manera el sujeto tatuado indique en qué partes de su cuerpo se encuentran ubicados los tatuajes y es posible corroborar si cuentan con el $10 \%$ del cuerpo tatuado.

En satisfacción corporal, el Multidimensional Body Self Relations Questionnaire (MBSRQ) es una escala que consta de 45reactivos encargados de evaluar los aspectos actitudinales respecto al constructo "imagen corporal" involucrando los aspectos cognitivos y conductuales del mismo. Esta prueba se aplica desde los quince años de edad y consta de manual y hoja de respuesta. Para esta investigación se contó con la versión adaptada al español por Botella, Ribas y Benito (2009), siendo sometida a un proceso de validez de constructo previo a las aplicaciones.

\section{Procedimiento}

Posterior al consentimiento informado y aprobados los criterios de inclusión, se suministraron los formatos con el dibujo del esquema corporal y la escala MBSRQ. Los datos obtenidos se procesaron con el paquete estadístico SPSS versión 19.

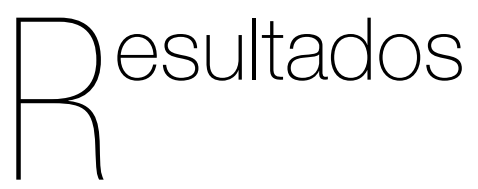

La prueba MBSRQ cuenta con un resultado general y un total de 4 sub escalas que permiten identificar la satisfacción de la imagen corporal en el sujeto. Siendo así, las variables arrojadas por la prueba son Satisfacción con la Imagen Corporal (SIC), Importancia Subjetiva de la Corporalidad (ISC), Conductas Orientadas a Mantener la Forma Física (COMF), Atractivo Físico Autoevaluado (AFA), y Cuidado del Aspecto Físico (CAF).

Con el fin de identificar cual era la distribución de las variables del MBS$R Q$, se utilizó la prueba estadística de Kolmogorov-Sminov del paquete estadístico SPSS versión 19 visualizada en la Tabla 1. Los resultados indican que las variables SIC, ISC, AFA, Y CAF no son normales, pero COMF tiene distribución normal.

Tabla 1

Resultados de la prueba de Kolmogorov-sminov para todas las escalas.

\begin{tabular}{cccccc}
\hline \multicolumn{7}{c}{ Prueba de Kolmogorov-Smirnov para una muestra } \\
\hline & $\begin{array}{c}\text { Satisfacción } \\
\text { con la imagen } \\
\text { corporal (SIC) }\end{array}$ & $\begin{array}{c}\text { Importancia } \\
\text { subjetiva de la } \\
\text { corporalidad (ISC) }\end{array}$ & $\begin{array}{c}\text { Conductas } \\
\text { Orientadas a } \\
\text { Mantener la Forma } \\
\text { Física (COMF) }\end{array}$ & $\begin{array}{c}\text { Atractivo Físico } \\
\text { Autoevaluado } \\
\text { (AFA) }\end{array}$ & $\begin{array}{c}\text { Cuidado del As- } \\
\text { pecto Físico (CAF) }\end{array}$ \\
\hline Z de Kolmogorov-Smirnov & 1.936 & 2.086 & 1.197 & 1.570 & 2.030 \\
Sig. asintót. (bilateral) & .001 & .000 & .114 & .014 & .001 \\
\hline
\end{tabular}


Luego de determinar las distribuciones entre escalas, se realizó una distribución general frente a los resultados de satisfacción de la imagen corporal en ambos grupos (tatuados y no tatuados), para esto se realizó una figura de diagrama de caja (Figura 1) para evidenciar dichos puntajes.

En la Figura 1 se observa que existen diferencias en las puntuaciones de los participantes, siendo más elevadas en las personas con tatuajes que el grupo sin tatuarse. Con el fin de determinar si dichas diferencias son significativas, se procede a determinar los estadísticos pertinentes tanto en las variables con distribución normal (Conductas Orientadas a Mantener la Forma Física (COMF) como las variables no normales (satisfacción con la imagen corporal (SIC), Importancia subjetiva de la corporalidad (ISC), Atractivo Físico Autoevaluado (AFA) y Cuidado del Aspecto Físico (CAF).

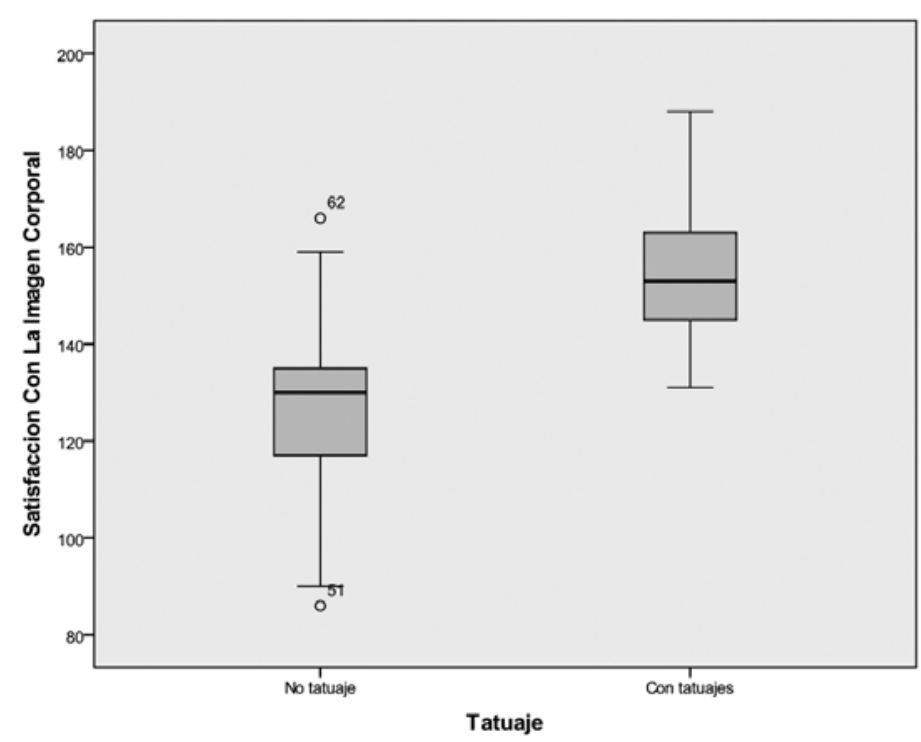

Figura 1. Gráfica de caja SIC general. Distribución de las puntuaciones en Satisfacción con la imagen corporal, según grupos de participantes con o sin tatuajes.

Para analizar la variable que tiene distribución normal (COMF) se utilizó la prueba $T$ para muestras independientes. La Tabla 2 muestra la prueba Levene que permite identificar si se asumen o no las varianzas de ambos grupos. Puesto que el dato evidencia que la significancia es de .951, se procede a analizar la prueba $T$ donde no se asumen varianzas iguales (Tabla 3).
Tabla 2.

Resultados prueba de Levene para varianzas de grupos

\begin{tabular}{cccc}
\hline & & \multicolumn{2}{c}{$\begin{array}{c}\text { Prueba de Levene para } \\
\text { la igualdad de varianzas }\end{array}$} \\
\hline & & $\mathrm{F}$ & Sig. \\
\hline Conductas & $\begin{array}{c}\text { Se han } \\
\text { Orientadas a }\end{array}$ & & \\
$\begin{array}{c}\text { Mantener la Forma } \\
\text { Fásica (COMF) }\end{array}$ & $\begin{array}{c}\text { varianzas } \\
\text { iguales }\end{array}$ & .004 & .951 \\
\hline
\end{tabular}


La prueba $T$ de la Figura 3, muestra una significancia de .000 lo que permite observar que los puntajes de participantes tatuados son significativamente diferentes frente al de participantes sin modificación corporal en relación a las Conductas Orientadas a Mantener la Forma Física (COMF), mostrando puntuaciones más altas en el grupo de estudiantes con más del $10 \%$ del cuerpo tatuado. Ahora, para verificar si la diferencia entre los grupos de personas con tatuajes y sin tatuajes en las variables restantes (con distribución no paramétrica) son significativas, se presentan (Tabla 4) los resultados del estadístico $U$ de Mann Whitney para muestras no relacionadas.

Tabla 3.

Resultados prueba T para sub-escala COMF

\begin{tabular}{|c|c|c|c|c|c|c|c|}
\hline \multicolumn{8}{|c|}{ Prueba de muestras independientes } \\
\hline & & \multirow{2}{*}{$\mathrm{t}$} & \multirow{2}{*}{$\mathrm{Gl}$} & \multirow{2}{*}{$\begin{array}{l}\text { Significancia. } \\
\text { (bilateral) }\end{array}$} & \multirow{2}{*}{$\begin{array}{l}\text { Error típ. de } \\
\text { la diferencia }\end{array}$} & \multicolumn{2}{|c|}{$\begin{array}{l}\text { 95\% Intervalo de confianza } \\
\text { para la diferencia }\end{array}$} \\
\hline & & & & & & Inferior & Superior \\
\hline $\begin{array}{l}\text { Conductas } \\
\text { Orientadas a } \\
\text { Mantener la Forma } \\
\text { Física (COMF) }\end{array}$ & $\begin{array}{l}\text { No se han } \\
\text { asumido varianzas } \\
\text { iguales }\end{array}$ & 7.378 & 65.261 & .000 & 1.029 & 5.534 & 9.642 \\
\hline
\end{tabular}

En la Tabla 4 se evidencia que con altos niveles de significancia, las personas con más del 10\% del cuerpo tatuado tienen mayores puntuaciones en las variables Importancia subjetiva de la Corporalidad
(ISC), Atractivo Físico Autoevaluado (AFA), Cuidado del Aspecto Físico (CAF) y Satisfacción con la Imagen Corporal (SIC) en universitarios tatuados y modificados corporalmente.

Tabla 4.

Resultados prueba $U$ de Mann-Whitney para muestras no relacionadas

\begin{tabular}{ccccc}
\hline & $\begin{array}{c}\text { Estadísticos de contraste } \\
\text { Importancia } \\
\text { subjetiva de la } \\
\text { corporalidad (ISC) }\end{array}$ & $\begin{array}{c}\text { Atractivo Físico } \\
\text { Autoevaluado (AFA) }\end{array}$ & $\begin{array}{c}\text { Cuidado del Aspecto } \\
\text { Físico (CAF) }\end{array}$ & $\begin{array}{c}\text { Satisfacción con la } \\
\text { imagen corporal }\end{array}$ \\
\hline U de Mann-Whitney & 289.000 & 709.500 & 944.500 & 229.500 \\
Sig. asintót. (bilateral) & .000 & .000 & .006 & .000 \\
\hline
\end{tabular}

a. Variable de agrupación: Tatuaje

\section{Resultados según distribución por sexo}

Una vez identificados los resultados generales, es importante describir si existe una diferencia significativa entre la satisfacción de la Imagen Corporal de hombres y mujeres tatuados frente a hombres y mujeres sin modificaciones corporales, para esto se procede a realizar gráficas de las pruebas 
no paramétricas (COMF) y gráficas de las pruebas paramétricas (SIC, ISC, AFA, Y CAF) así como sus respectivos estadísticos.

\section{Conductas Orientadas a Mantener la Forma Física (COMF)}

La escala conductas orientadas a mantener la forma física se relaciona con los comportamientos enfocados a mantener un buen estado físico, cuidar de la salud del sujeto y mantenerse atractivo así como la frecuencia de los mismos. En la figura 2 se evidencia la distribución de puntajes frente a hombres tatuados y no modificados corporalmente, los datos muestran que existen diferencias entre ambos grupos encontrando más elevados los puntajes de hombres tatuados. Ahora, la Figura 3 muestra la distribución de puntajes entre mujeres tatuadas y no modificadas corporalmente, se evidencian mayores niveles de conductas orientadas a mantener la forma física en el grupo de mujeres tatuadas.

Luego, para identificar si las diferencias entre ambos grupos de sexo son significativas, se procede a realizar prueba $T$ para muestras paramétricas evidenciada en la Tabla 5 donde se evidencia que con altos niveles de significancia existen diferencias entre las conductas orientadas a mantener la conducta física teniendo mayores niveles los hombres tatuados frente a hombres no modificados corporalmente, así como en mujeres tatuadas respecto a las no modificadas corporalmente.

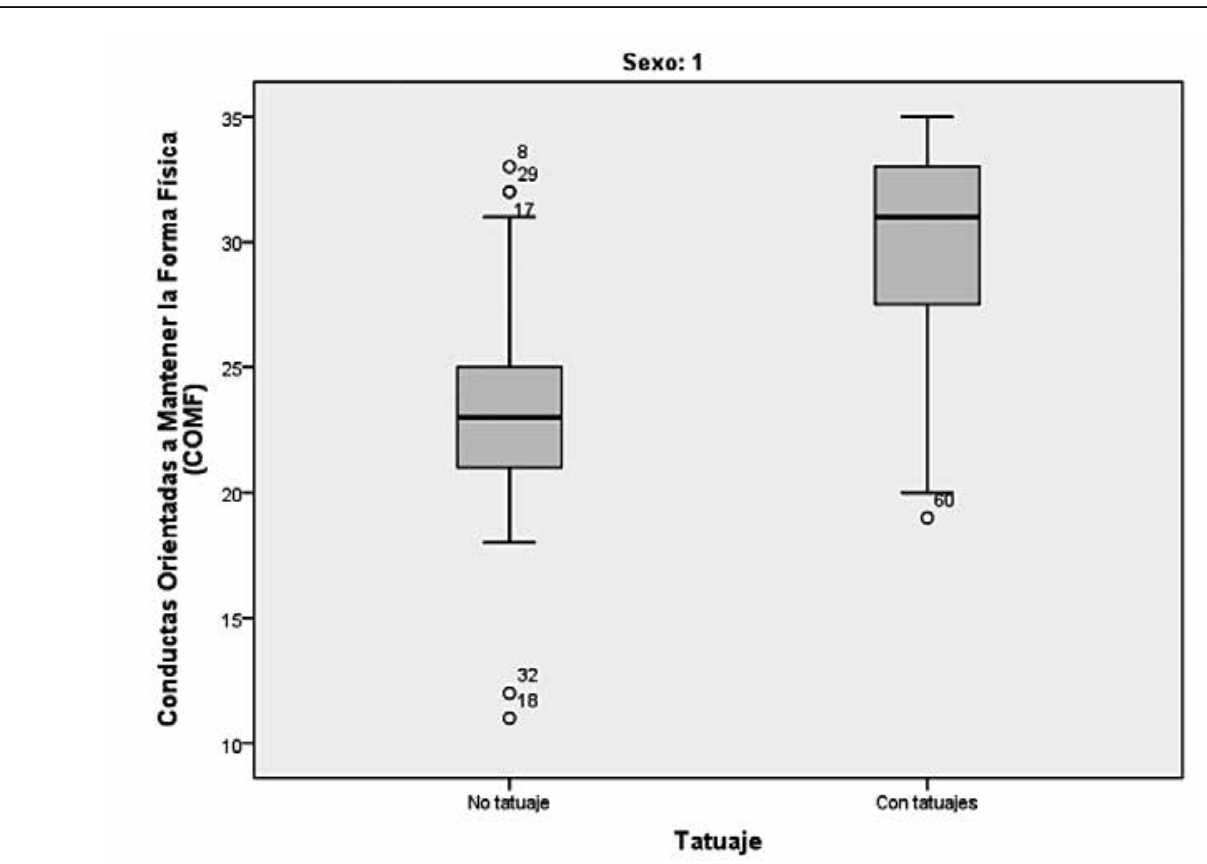

Figura 2. Gráfico de caja COMF hombres. Distribución de las puntuaciones en Conductas Orientadas a Mantener la Forma Física en hombres con o sin tatuajes. 


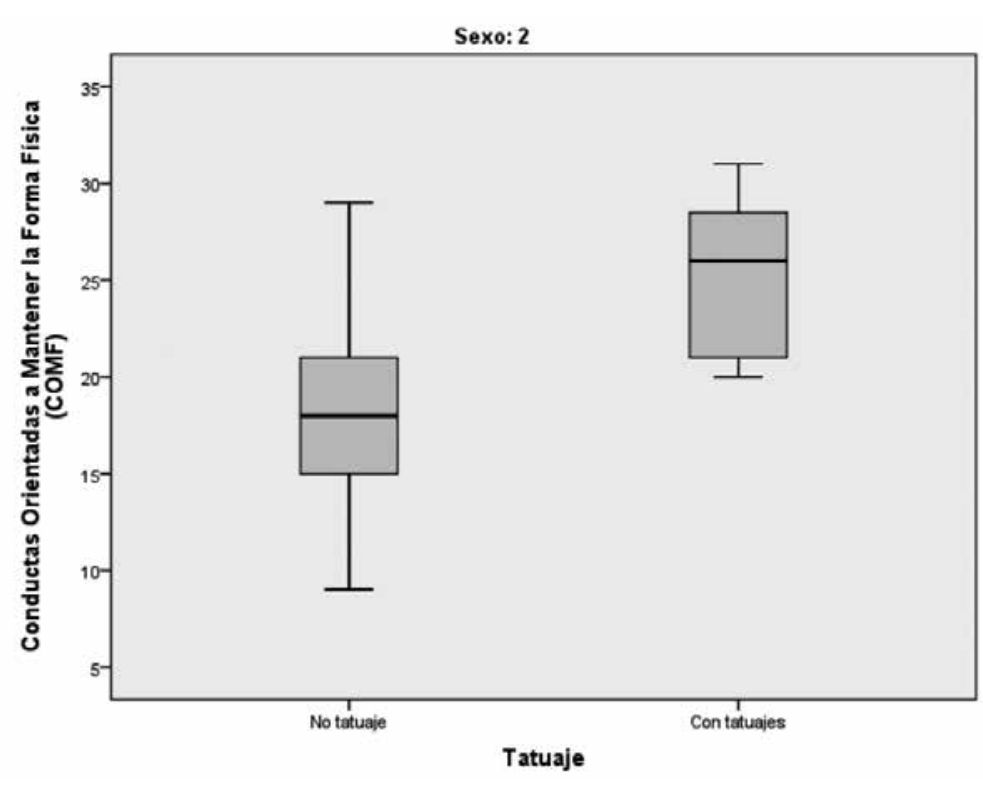

Figura 3. Gráfico de caja COMF mujeres. Distribución de las puntuaciones en Conductas Orientadas a Mantener la Forma Física en mujeres con o sin tatuajes.

Tabla 5.

Resultados de prueba T para muestras independientes COMF

\begin{tabular}{|c|c|c|c|c|c|}
\hline \multicolumn{6}{|c|}{ Prueba T para la igualdad de medias } \\
\hline & Sexo & & $\mathrm{T}$ & gl & Sig. (bilateral) \\
\hline \multirow{2}{*}{1} & \multirow{2}{*}{$\begin{array}{l}\text { Conductas Orientadas } \\
\text { a Mantener la For- } \\
\text { ma Física (COMF) }\end{array}$} & $\begin{array}{l}\text { Se han asumido va- } \\
\text { rianzas iguales }\end{array}$ & -5.138 & 62 & .000 \\
\hline & & $\begin{array}{c}\text { No se han asumido } \\
\text { varianzas iguales }\end{array}$ & -5.042 & 43.186 & .000 \\
\hline \multirow{2}{*}{2} & \multirow{2}{*}{$\begin{array}{l}\text { Conductas Orientadas } \\
\text { a Mantener la For- } \\
\text { ma Física (COMF) }\end{array}$} & $\begin{array}{l}\text { Se han asumido va- } \\
\text { rianzas iguales }\end{array}$ & -5.031 & 50 & .000 \\
\hline & & $\begin{array}{c}\text { No se han asumido } \\
\text { varianzas iguales }\end{array}$ & -5.261 & 16.815 & .000 \\
\hline
\end{tabular}

A continuación se encuentran las gráficas y pruebas para identificar diferencias de las sub escalas no paramétricas.

\section{Satisfacción Con La Imagen Corporal (SAC)}

La escala de satisfacción con la imagen corporal es la suma general de
39 ítems del test MBSRQ los cuales contemplan todas las características del cuidado y satisfacción de imagen corporal. La Figura 4 a continuación expuesta, muestra las diferencias de satisfacción corporal entre hombres tatuados y hombres no modificados corporalmente, los datos muestran mayor puntuación en hombres con el $10 \%$ del cuerpo tatuado. 
Por su parte la Figura 5 muestra la diferencia de puntuaciones entre mujeres con tatuajes y no modificadas corporalmente respecto a la satisfacción con su imagen corporal. Los datos expresan que las mujeres tatuadas tienen mayores niveles en esta escala.
Luego de obtener los datos expresados en las gráficas, se realizó prueba estadística con el fin de conocer si existen diferencias significativas entre los grupos, para tal objetivo se implementó prueba de Mann-Whitney para muestras no paramétricas expresada en la Tabla 6.

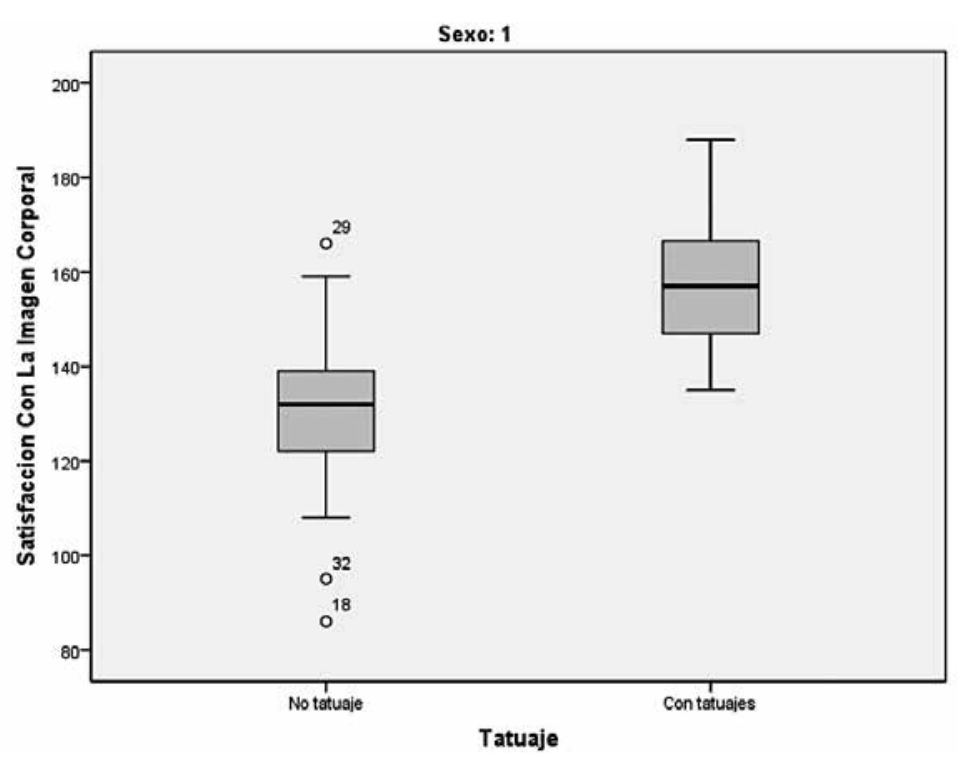

Figura 4. Gráfico de caja SIC hombres. Distribución de las puntuaciones en satisfacción con la imagen corporal de hombres con o sin tatuajes.

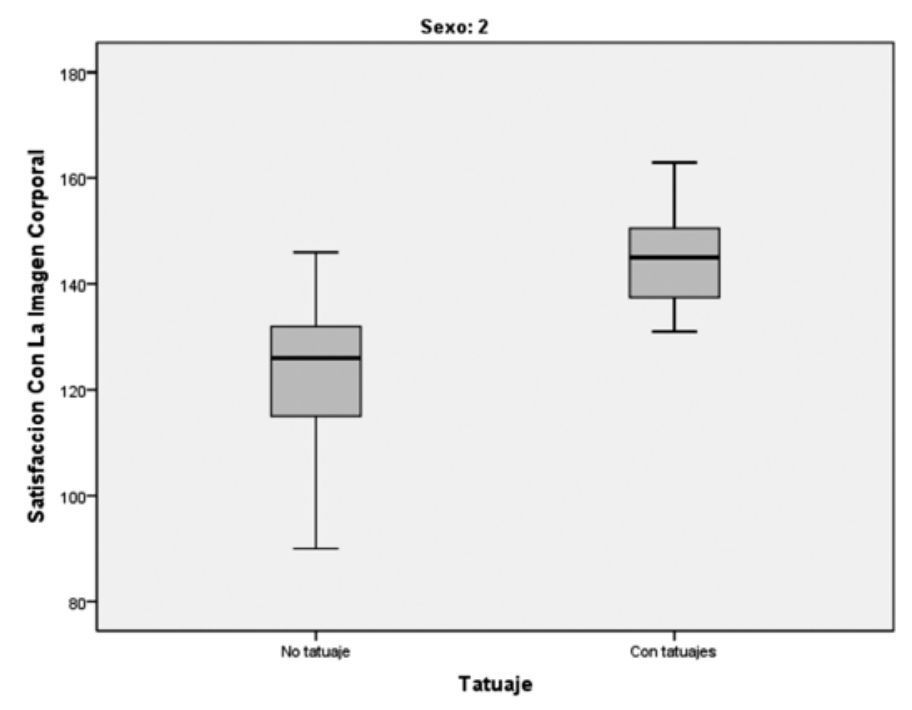

Figura 5. Gráfico de caja SIC mujeres. Distribución de las puntuaciones en satisfacción con la imagen corporal de mujeres con o sin tatuajes. 
Tabla 6.

Resultados prueba $U$ de Mann-Whitneysubescala SAC.

\begin{tabular}{ccc}
\hline & Sexo & $\begin{array}{c}\text { Satisfacción Con La } \\
\text { Imagen Corporal }\end{array}$ \\
\hline \multirow{2}{*}{ Hombres } & U de Mann-Whitney & 76.000 \\
& Sig. asintót. (bilateral) & .000 \\
\multirow{2}{*}{ Mujeres } & U de Mann-Whitney & 39.000 \\
& Sig. asintót. (bilateral) & .000 \\
\hline \multicolumn{3}{c}{ a. Variable de agrupación: Tatuaje } \\
\hline
\end{tabular}

La Tabla 6 muestra que con diferencias altamente significativas, los hombres con el $10 \%$ del cuerpo tatuado presentan mayor satisfacción con la imagen corporal frente a los hombres sin modificaciones corporales. De igual manera las mujeres tatuadas presentan mayores niveles de satisfacción con la imagen corporal diferenciándose significativamente del grupo de mujeres no modificadas corporalmente.

\section{Importancia subjetiva de la corporalidad (ISC)}

La importancia subjetiva de la corporalidad se refiere a ¿qué tan importante es para un sujeto su aspecto? $Y$ ¿qué relevancia tiene este aspecto en la vida del mismo? En la Figura 6 se expresan los resultados en hombres tatuados y no modificados corporalmente frente a la importancia subjetiva de la corporalidad, se hace evidente que los hombres tatuados expresan mejores resultados además de una mejor distribución del puntaje.

En la Figura 7 se encuentran los resultados de mujeres tatuadas frente a las no modificadas corporalmente, los datos expresan que hay mayor nivel de importancia subjetiva de la corporalidad en aquellas mujeres que tienen el 10\% del cuerpo tatuado. Luego, para corroborar si las diferencias halladas en las gráficas son significativas, se realizó prueba de Mann-Whitney para pruebas no paramétricas, esta se evidencia en la Tabla 7 mostrando que con diferencia significativa de .000 las mujeres y hombres tatuados manifiestan mayor satisfacción con la imagen corporal frente a mujeres y hombres que no poseen ninguna modificación corporal.

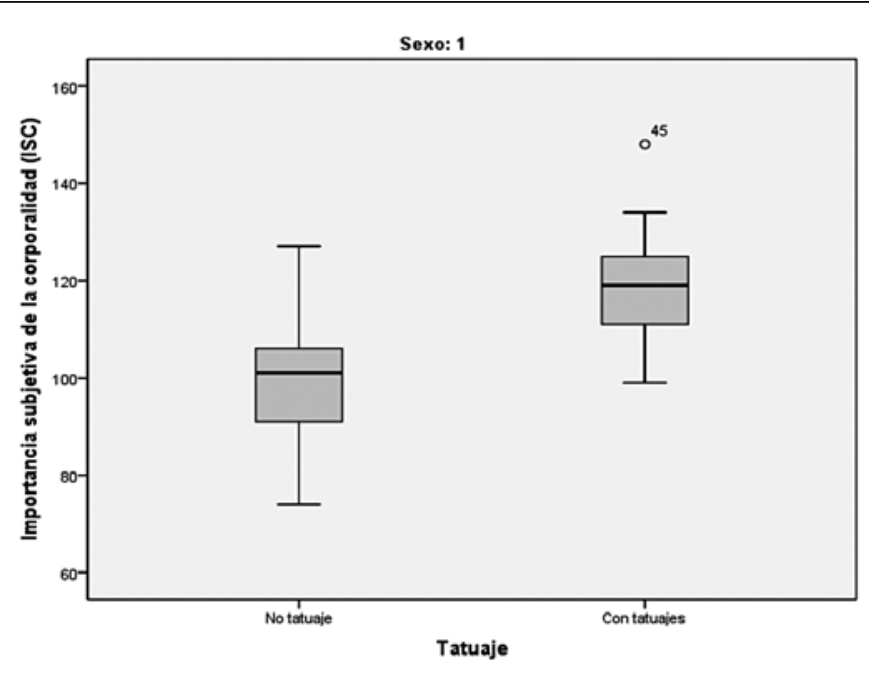

Figura 6. Gráfico de caja ISC hombres. Distribución de las puntuaciones en importancia subjetiva de la corporalidad de hombres con o sin tatuajes. 


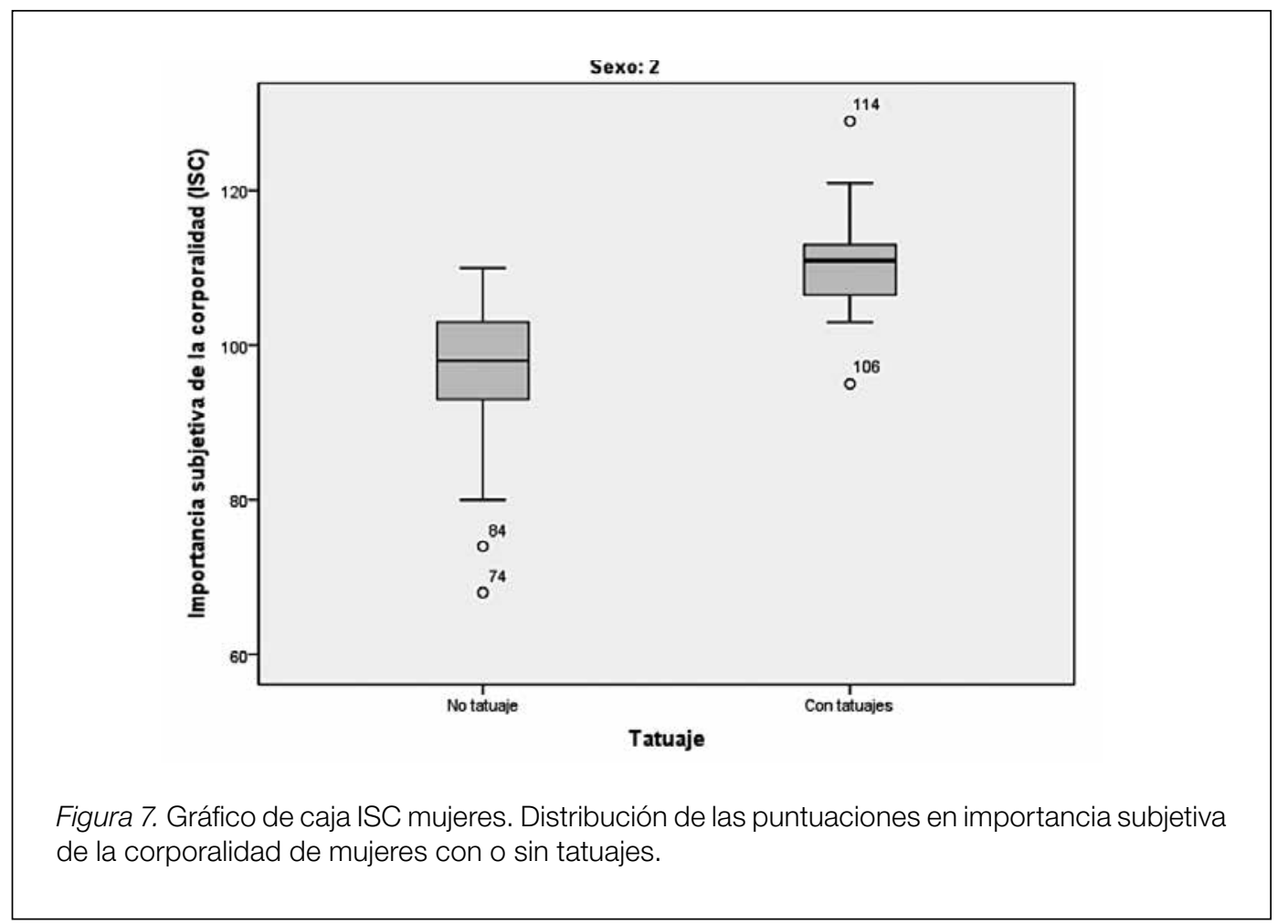

Tabla 7.

Resultados prueba $U$ de Mann-Whitney subescala ISC

\begin{tabular}{ccc}
\hline \multicolumn{3}{c}{ Estadísticos de contraste $^{\mathrm{a}}$} \\
\hline Sexo & $\begin{array}{c}\text { Importancia } \\
\text { subjetiva de la } \\
\text { corporalidad (ISC) }\end{array}$ \\
\hline Hombres & U de Mann-Whitney & 108.500 \\
& Sig. asintót. (bilateral) & .000 \\
Mujeres & U de Mann-Whitney & 47.000 \\
& Sig. asintót. (bilateral) & .000 \\
\hline
\end{tabular}

\section{Atractivo Físico Autoevaluado (AFA)}

El atractivo físico se refiere a los juicios calificativos que tenían los participantes acerca de su propia corporalidad, cómo calificaban su aspecto y qué opinión tenían del mismo. En la figura 8 se muestran los puntajes de hombres con el 10\% del cuerpo tatuado y hombres sin ninguna modificación corporal frente al atractivo físico autoevaluado. Los resultados muestran que efectivamente los hombres tatuados presentan mayores niveles de puntuación en esta escala.

Por su parte, la figura 9 muestra los resultados del atractivo físico autoevaluado en mujeres que cuentan con $10 \%$ del cuerpo tatuado y mujeres que no se han realizado ninguna modificación corporal. Los datos expresan que existen diferencias entre el grupo de mujeres tatuadas y no tatuadas. 


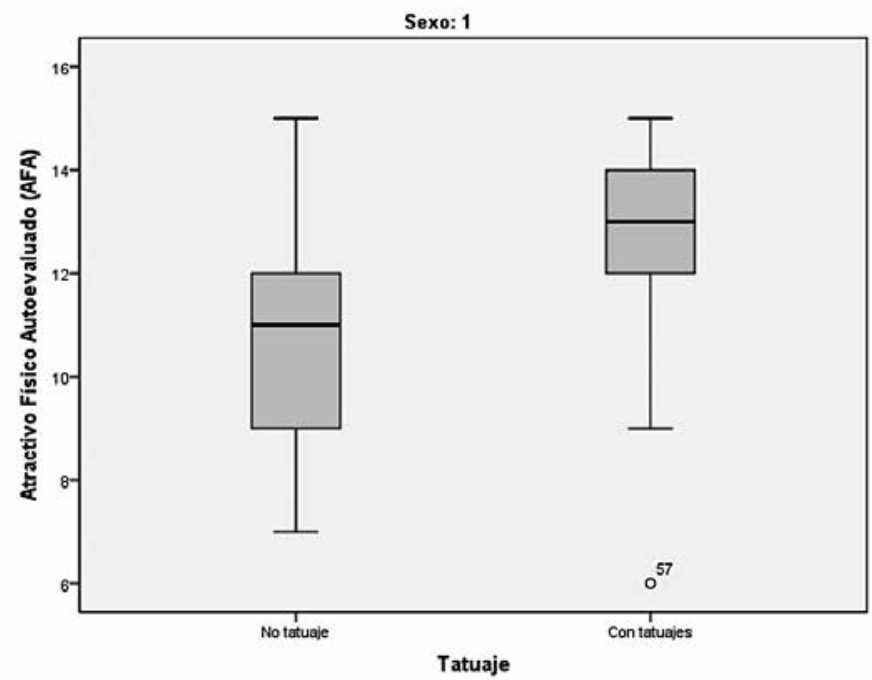

Figura 8. Gráfica de caja AFA hombres. Distribución de las puntuaciones en atractivo físico autoevaluado de hombres con o sin tatuajes.

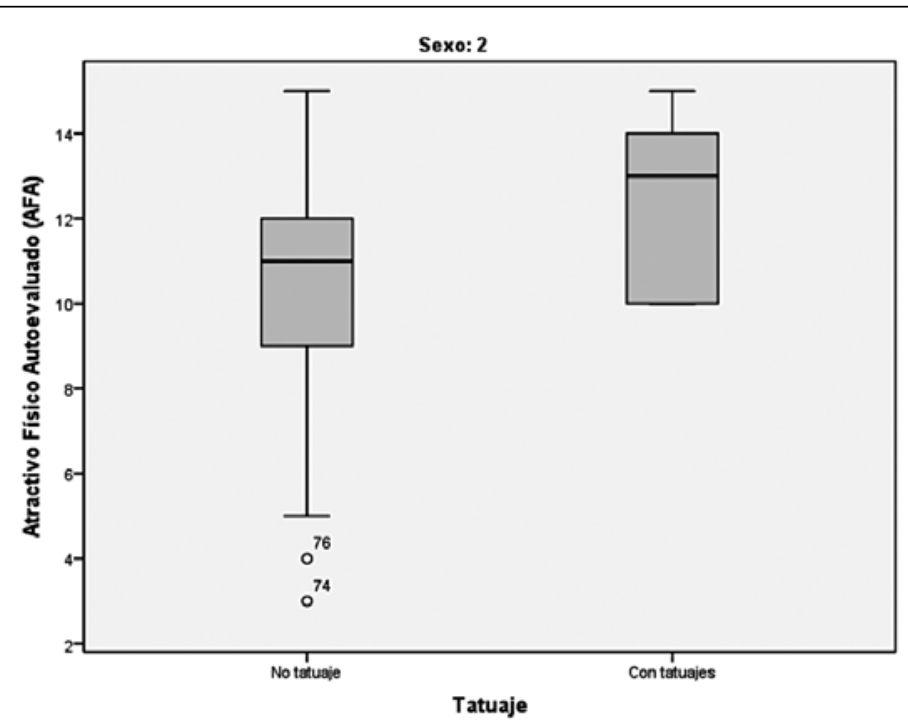

Figura 9. Gráfico de caja AFA mujeres. Distribución de las puntuaciones en atractivo físico autoevaluado de mujeres con o sin tatuajes.

Con el fin de identificar si las diferencias de las puntuaciones expresadas en las Gráficas 8 y 9 son de carácter significativo, se procedió a realizar la prueba de $U$ de Mann-Whitney (Tabla 8) para pruebas no paramétricas. Los resultados de la Tabla 8 muestran que el grupo de hombres tatuados y no modificados corporalmente presentan diferencias altamente significativas, siendo los tatuados quienes presentan mayores niveles de atractivo físico autoevaluado. 
Tabla 8.

Resultados prueba $U$ de Mann-Whitney subescala AFA

\begin{tabular}{ccc}
\hline Sexo & & $\begin{array}{c}\text { Atractivo Físico } \\
\text { Autoevaluado (AFA) }\end{array}$ \\
\hline Hombres & U de Mann-Whitney & 203.000 \\
& Sig. asintót. (bilateral) & .000 \\
Mujeres & U de Mann-Whitney & 143.000 \\
& Sig. asintót. (bilateral) & .063 \\
\hline
\end{tabular}

A pesar de que la Figura 9 expresa diferencias entre el grupo de mujeres tatuadas y las no modificadas corporalmente, lo estadísticos de la Tabla 8 manifiestan que esta diferencia no es significativa, por lo tanto el atractivo físico autoevaluado entre los dos grupos de mujeres no es significativamente diferente.

\section{Cuidado Del Aspecto Físico (CAF)}

La sub escala cuidado del aspecto físico se refiere a la atenciones y comportamientos orientados a mantener una adecuada apariencia; la figura 10 presenta los resultados en esta escala respecto a hombres que cuentan con $10 \%$ del cuerpo tatuado y hombres sin ninguna modificación corporal. Los resultados muestran que los hombres tatuados obtienen mayores puntuaciones frente al cuidado de su aspecto físico.

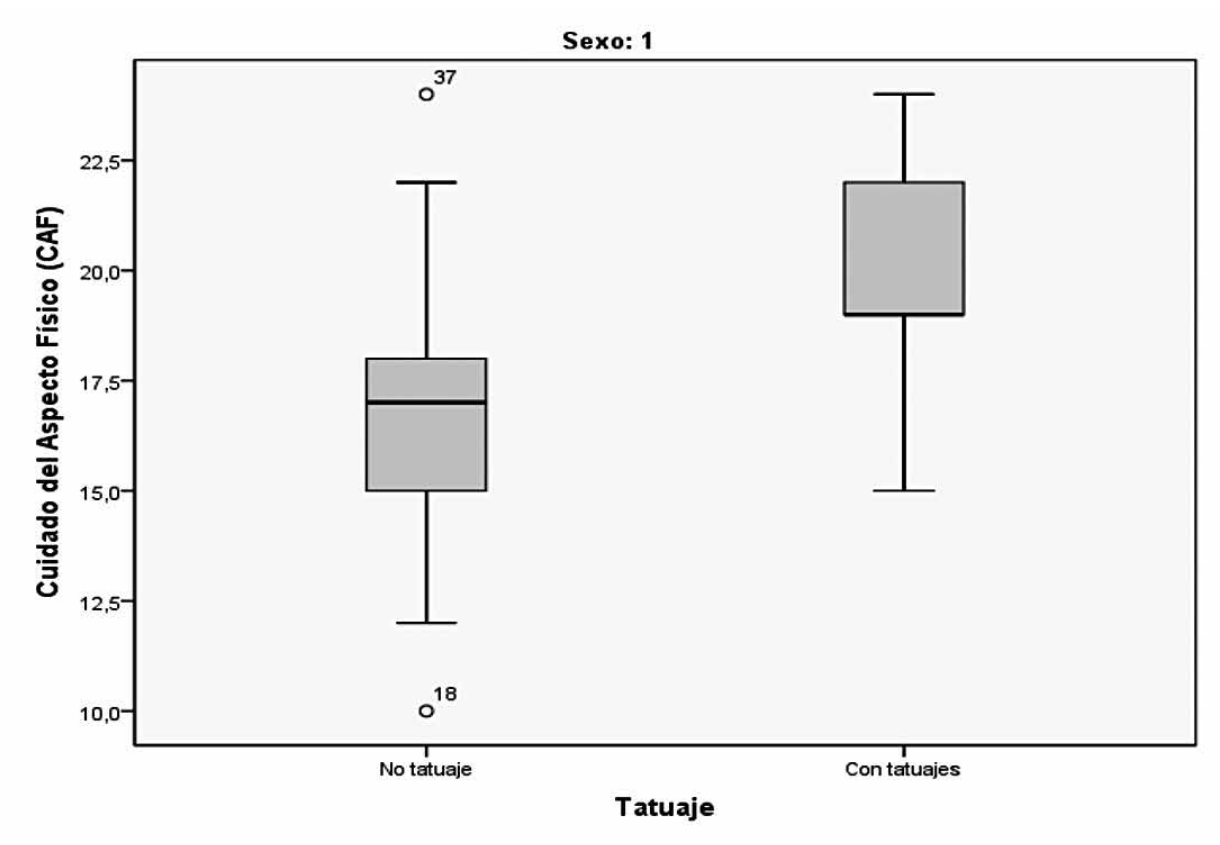

Figura 10. Gráfico de caja CAF hombres. Distribución de las puntuaciones en cuidado del aspecto físico de hombres con o sin tatuajes. 


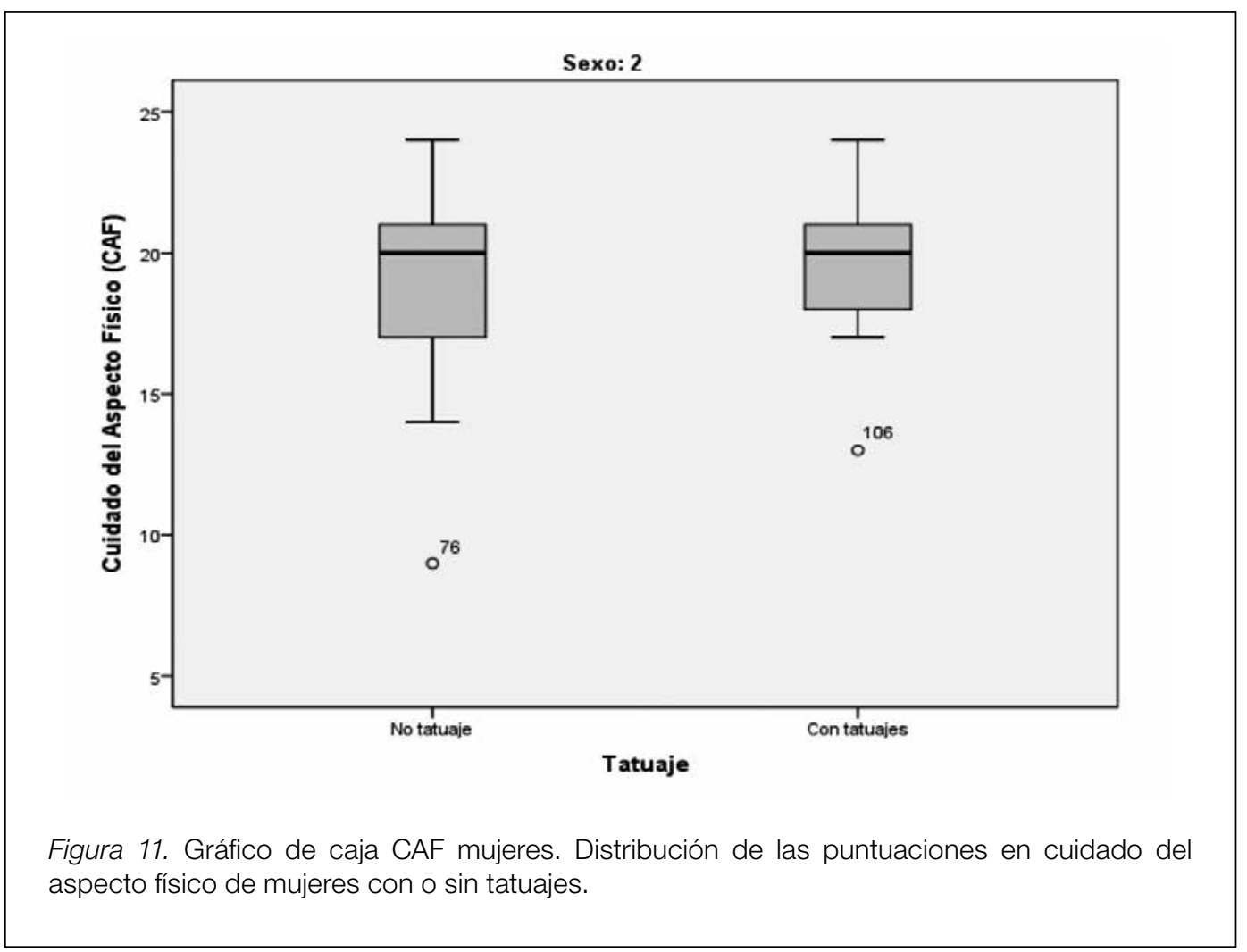

En la Figura 11 se presentan las distribuciones de puntuación en mujeres tatuadas y las no modificadas corporalmente frente a su cuidado del aspecto físico. La grafica muestra que no hay diferencias de puntuación entre ambos grupos. Con el fin de identificar las diferencias estadísticas entre ambos grupos se realizó prueba de MannWhitney para pruebas no paramétricas, los resultados se muestran en la Tabla 9. Los resultados estadísticos muestran que los hombres que cuentan con el $10 \%$ del cuerpo tatuado presentan resultados diferentemente significativos respecto a los no modificados corporalmente. Frente al grupo de mujeres tatuadas y no modificadas corporalmente se corrobora que efectivamente no existen diferencias entre su cuidado del aspecto físico.
Tabla 9.

Resultados prueba $U$ de Mann-Whitney subescala CAF

\begin{tabular}{ccc}
\hline Sexo & & $\begin{array}{c}\text { Cuidado del As- } \\
\text { pecto Físico (CAF) }\end{array}$ \\
\hline Hombres & U de Mann-Whitney & 207.500 \\
& Sig. asintót. (bilateral) & .000 \\
Mujeres & U de Mann-Whitney & 206.500 \\
& Sig. asintót. (bilateral) & .668 \\
\hline
\end{tabular}

Para finalizar los resultados, se decidió realizar estadísticos de fiabilidad para la prueba MBSRQ con el fin de generar mayor seguridad en las puntuaciones obtenidas; por lo tanto se procedió a realizar Alfa de Cronbach para analizar los ítems en relación al sexo, estos resultados se muestran en la Tabla 10. Además de ello se realizó Alfa de Cronbach general de la prueba evidenciado en la Tabla 11. 
Los resultados de las Tablas 10 y 11 muestran puntajes altos de fiabilidad en el test, lo cual permite generar mayor seguridad en los hallazgos logrados a través del mismo.

Tabla 10.

Resultados Alfa de Cronbach por sexo.

\begin{tabular}{ccc}
\hline \multicolumn{3}{c}{ Estadísticos de fiabilidad } \\
\hline Sexo & Alfa de Cronbach & N de elementos \\
\hline Hombres & .908 & 45 \\
Mujeres & .827 & 45 \\
\hline
\end{tabular}

Tabla 11.

Resultados Alfa de Cronbach general

\begin{tabular}{cc}
\hline \multicolumn{2}{c}{ Estadísticos de fiabilidad } \\
\hline Alfa de Cronbach & Número de elementos \\
\hline .887 & 45 \\
\hline
\end{tabular}

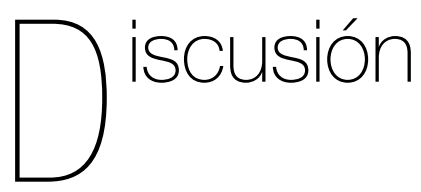

A partir de los resultados obtenidos en esta investigación se evidencia que efectivamente el grupo de universitarios que cuentan con el 10\% del cuerpo tatuado se encuentran más satisfechos con su imagen corporal a diferencia del grupo de universitarios que no tienen ningún tipo de modificación corporal. Debido a que la población sin modificaciones corporales y la población de sujetos tatuados no tenían antecedentes psicológicos o psiquiátricos que relacionaran con un trastorno metal, se plantea que el puntaje de satisfacción con la imagen corporal no está relacionada a variables de alteración cognitiva o patológica, además, los individuos tatuados requerían no ser parte de alguna sub cultura o tribu urbana que pudiese influir en la adquisición de sus marcas corporales; por lo tanto, la posible diferencia entre ambos grupos es el tatuaje propiamente dicho. A partir de ello se propone que el tatuaje genera un efecto en el sujeto que beneficia directamente la percepción de la imagen corporal, esto en medida que los resultados muestran a los individuos tatuados con mayores niveles de atractivo físico autoevaluado referido al juicio valorativo que tienen de su propio aspecto (AFA), cuidados que implementan a mantener su aspecto físico (CAF), importancia que le dan a su corporalidad (ISC) y conductas orientadas a mantener de manera adecuada el aspecto físico (COMF). Se sugiere realizar futuros estudios donde se tenga en cuenta el estrato socio económico de los participantes disminuyendo la probabilidad de variables influyentes en los resultados.

Investigaciones como las de Crerand, Cash y Whitaker, (2006) han demostrado que existen cambios de la satisfacción de la imagen corporal en sujetos que han sido expuestos a una cirugía plástica; para esta investigación, es el tatuaje a diferencia de la cirugía plática la técnica que muestra un aumento en la satisfacción de la imagen corporal generando diferencias significativas entre la población que ha recurrida a esta y la que no. Es importante señalar que esta medición no tuvo en cuenta el tiempo en el que se hubiesen tatuado los participantes, por tal razón se sugiere realizar estudios pre y post en los que se evalué la satisfacción de la imagen corporal antes y después de realizarse un tatuaje.

Por otro lado, nuestros resultados diferencian a los resultados hallados 
por Bolton, Pruzinsky, Cash y Persing, (2003) donde personas que se sometieron a una cirugía plástica presentaron de manera residual conductas autodestructivas y depresión; en esta investigación se evidencia que el sujeto tatuado se siente satisfecho con su aspecto, se preocupa por el mismo y no ha tenido necesidad de tratar algún trastorno de carácter mental.

Referente a la distribución por sexo, la teoría sugiere que es mayor el número de mujeres que presenta insatisfacción con la imagen corporal; investigaciones como las de Fernández, Otero, Castro y Prieto (2003) describen que las mujeres presentan mayores niveles de preocupación por su peso y mantener dietas lo cual se asocia directamente a insatisfacción con su imagen corporal. En nuestro estudio se describe que si bien las mujeres tatuadas también presentan mayor número de comportamientos relacionados a mantener un adecuado peso e implementar dietas (escala ISC), estos no se relacionan con insatisfacción de su imagen corporal como si sucede con el grupo de mujeres que no poseen ninguna modificación corporal. Es decir, se corrobora que las mujeres tatuadas también se preocupan por mantener un favorable aspecto físico a través de conductas de mantenimiento, pero a diferencia de la teoría anteriormente descrita las mujeres tatuadas se encuentran más satisfechas con su imagen corporal.

Por otro lado, las mujeres tatuadas presentaron niveles de puntuación más alto en las escalas importancia subjetiva de la corporalidad, comportamientos orientados a mantener la forma física y satisfacción con la imagen corporal, pero referente a atractivo físico autoevaluado y cuidado del aspecto físico no se evidenciaron diferencias significativasen relación a las mujeres sin modificaciones corporales. Esto indica que tanto mujeres tatuadas y no modificadas corporalmente suelen describirse y calificarse positivamente cuándo se les indaga al igual que suelen orientar comportamientos constantes para mejorar su aspecto físico; la diferencia radica en un aspecto de orden intrínseco donde las mujeres tatuadas mostraron sentirse más satisfechas con su imagen corporal y a otorgarle gran importancia a este aspecto diferenciándose de aquellas que no se han realizado ninguna modificación corporal. A pesar de que los estadísticos evidencian una adecuada distribución, se sugiere que en futuros estudios la muestra de mujeres tatuada sea lo más extensa posible, favoreciendo los procesos de generalización en la población.

Frente a la población masculina, se evidencia que los sujetos con el 10\% del cuerpo tatuado presentan resultados significativamente diferentes en relación a los hombres sin modificaciones corporales. Por un lado son los hombres tatuados quienes muestran mayor satisfacción de la imagen corporal y mayor número de comportamientos para cuidar su aspecto físico. Literatura como la propuesta por Ibarzabal y Tubio (2008) menciona que hombres fisiculturistas suelen presentar mayores comportamientos relacionados al cuidado de su cuerpo en relación de quienes no son competidores, es nuestra investigación se evidencia que son los hombres tatuados quienes realizan mayor número de comportamientos para cuidar el aspecto físico (COMF) como 
es practicar deporte, hacer ejercicio, aumentar la fuerza física y estar activo durante el año en relación de aquellos que no se han modificado su cuerpo. Como investigación futura, es pertinente comparar grupos de hombres tatuados y no tatuados que asistan al gimnasio con el fin de identificar diferencias o similitudes en relación a la percepción de su imagen corporal.

En nuestro estudio la población de hombres y mujeres con el 10\% del cuerpo tatuado se diferencian en número de muestra, por lo tanto no se realizan estadísticos para identificar relaciones de imagen corporal entre estos dos grupos. Siendo así, se sugiere realizar estudios próximos donde se analice la diferencia de satisfacción en imagen corporal frente a hombres y mujeres que tengan el $10 \%$ del cuerpo tatuado detallando en que sexo existe un efecto mayormente significativo del tatuaje.

Por último es importante referirse a la satisfacción de los sujetos tatuados como un beneficio en contexto; es decir, si el sujeto tatuado muestra mayor satisfacción de la imagen corporal y de esta manera hay probabilidad de disminuir comportamientos de evitación, trastornos de la imagen corporal o dificultades para las relaciones interpersonales, esto quiere decir que existe funcionalidad en términos psicológicos para el individuo y esto podría ser una de las razones por la cual existe mayor demanda de este fenómeno en la actualidad. Aun así es indispensable realizar réplicas de esta investigación contando con mayor número participantes tatuados que permitan corroborar o reevaluar los datos obtenidos en esta investigación.

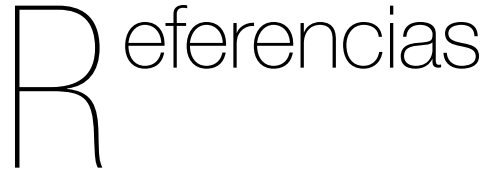

Aafjes, M. (2008). Belleza Producida y Campos Maleables. Buenos Aires. Facultad Latinoamericano de Ciencias Sociales (FLACSO), Online, recuperado el 20 de Abril de http://flacsoandes.org/ dspace/bitstream/10469/1159/1/Tesis_ Marieke_Aafjes.pdf

Álvarez, N. (2000). Tatuajes. Red de Educación del Consumidor, Guía didáctica del profesor, Online, Recuperado el 24 de Abril de http://www.kontsumobide. euskadi.net/contenidos/informacion/kb_ pubs_mat_didac/es_material/adjuntos/ diana_profesor_tatuajes_es.pdf

Álvarez, G., Franco, K., López, X., Mancilla, J., \& Vázquez, R. (2009). Imagen corporal y trastornos de la conducta alimentaria. Revista de Salud Pública, 11(4), 568-578.

Argüello Peña, L., \& Romero Carrera, I. (2012). Trastorno De La Imagen Corporal. Reduca (Enfermería, Fisioterapia y Podología), 4(1). Recuperado el 15 septiembre de 2013 de: http://www.revistareduca.es/ index.php/reducaenfermeria/article/ viewFile/1005/1017

Atencio, P., Molina, Z., \& Rojas, L. (2008). Influencia Del Género Y La Percepción De La Imagen Corporal En Las Conductas Alimentarias De Riesgo En Adolescentes De Mérida. In Anales Venezolanos de Nutrición, 21(2), 85-90.

Ayensa, J., Martínez, K., \& Rancel, F. (2005). Alteración de la imagen corporal en un grupo de usuarios de gimnasios. 
Enseñanza e Investigación en Psicología, 10(1), 161-169.

Bolton, M., Pruzinsky, T., Cash, T., \& Persing, J. (2003). Measuring outcomes in plastic surgery: body image and quality of life in abdominoplasty patients. Plastic and Reconstructive Surgery, 112(2), 619-25.

Bosello, R., Favaro, A., Zanetti, T., Soave, M., Vidotto, G., Huon, G., \&Santonastaso, P. (2009). Tattoos and Piercings In Adolescents: Family Conflicts And Temperament. U.S. Rivista di Psichiatria, 45(2), 102-106.

Botella, L., Ribas, E., \& Benito, J. (2009) Evaluación psicométrica de la imagen corporal: Validación de la versión española del Multidimensional Body Self Relation Questionnaire (MBSRQ). Revista Argentina de Clínica Psicológica, 18 (3), $253-264$.

Busaniche, J., Eymann, A., Mulli, V., Paz, M., \& Catsicaris, C. (2006). Asociación entre adolescentes tatuados y conductas de riesgo. Archivos Argentinos de Pediatría, 104(4), 309-315.

Bruch, H. (1962). Perceptual And Conceptual Disturbances In Anorexia Nervosa. Obstetrical \& Gynecological Survey, 17(5), 730-732.

Carroll, S., Riffenburgh, R., Roberts, T.,\&Myhre, E. (2002). Tattoos And BodyPiercings As Indicators Of AdolescentRiskTakingBehaviors. Pediatrics, 109(6), 1021-1027.

Cash, T., \& Pruzinsky, T. (1990). Body images: Development, deviance, and change. Guilford Press, 21, 361.
Cassab, J. (2002). Psicopatología De La Expresión A Partir De Los Tatuajesen En Pacientes Psiquiatricos Internados - Un Estudio Epidemiológico. México, D.F.: Revista neurología, neurocirugía y psiquiatría. (2). Recuperado de http:// adminweb.dyndns.org/ijapsi/doctos/ librosYpub/CASSAB_tatuajes_2001.pdf

Castle, D., Honigman, R., \& Phillips, K. (2002). Does cosmetic surgery improve psychosocial wellbeing? Medical Journal of Australia, 176(12), 601-603.

Crerand, C., Cash, T., \& Whitaker, L. (2006). Cosmetic surgery of the face. Psychological aspects of reconstructive and cosmetic plastic surgery: Empirical, clinical, and ethical issues. Philadelphia: Lippincott Williams \&Wilkins, 233-249.

Cucarella, M. (2013). Bienestar emocional, imagen corporal, autoestima y sexualidad en mujeres con cáncer de mama. Psicooncología, 10(1), 199-200.

Danilla, S., Cuevas, P., Domínguez, C., Di silvestre, C., Soto, F., Silva, P., \& Sepúlveda, S. (2013). Medición de los resultados desde la perspectiva del paciente en cirugía de contorno corporal: creación del instrumento Body-QoL. Revista Chilena de Cirugía, 65(6), 495-501.

Espina, A., Ortego, M., Yenes, F., \& Alemán, A. (2001). La Imagen Corporal En Los Trastornos Alimentarios. Psicothema, 13(4), 533-538.

Faul, F. (2008). G*Power versión 3.0.10. [Paquete estadístico]. Universidad de Kiel: Alemania.

Fernández, M., Otero, M., Castro, Y., \& Prieto, M. (2003). Hábitos alimentarios e imagen 
corporal en estudiantes universitarios sin trastornos alimentarios. International Journal Clinical Health Psychology, 3(1), 23-33.

Gallego, F. (2010). Esquema Corporal E Imagen Corporal. Revista Española de Educación Física y Deportes, (12), 44-63.

Ganter, R. (2005). De Cuerpos, Tatuajes Y Culturas Juveniles. Espacio abierto, 15(1y2), 427-453.

Garner, D., \& Garfinkel, P. (1981). Body Image In Anorexia Nervosa: Measurement, Theory And Clinical Implications. The International Journal of Psychiatry in Medicine, 11(3), 263-284.

Hermosillo, G., Tovar, U., Gómez, J., Herrera, P., \& Sánchez-Mejoradaa, G. (2011). Clasificación De Tatuajes Como Método De Identificación Forense. Estudios de Antropología Biológica, 15(1), 219 - 227.

Hernández, R., Fernández, C. \& Baptista, P. (2010). Metodología de la investigación. Mac Graw Hill: México.

Hoyos, M., Jaramillo, C., Molina, E., Valverde, S., \& Posso, C. (2007). Evaluación de la superficie corporal quemada en pacientes del Hospital Universitario San Vicente de Paúl, Medellín. latreia, 20(1), 21.

Hurtado, D. (2008). Corporeidad y motricidad: Una forma de mirar los saberes del cuerpo. Revista Brasilera Educacao \& Sociedade, 29(102).

Ibarzabal, F., \& Tubio J. (2008).Imagen corporal en varones fisicoculturistas. Acta Colombiana de Psicología, 11(1), 75-88.
Jacobson, W., Edgerton, M., Meyer, E., Canter, A., \& Slaughter, R. (1960). Psychiatric evaluation of male patients seeking cosmetic surgery. Plastic and Reconstructive Surgery, 26(4), 356-372.

Le Breton, D. (2002). La sociología del cuerpo. Nueva Visión. Online, recuperado el 20 de Abril de 2014 de http://www. bioenergeticalatam.com.ar/docus/ LeBreton.pdf

López, R. (2007). Cuerpos Transgresores/ Cuerpos transgredidos: Carne y memoria marcadas. Los jóvenes y sus prácticas de modificación corporal. Última Década, 15(26), 103-119.

Manca, M. (2011). Agresiones Al Cuerpo En La Adolescencia ¿Redefinición De Los Límites Del Cuerpo ODesafíoEvolutivo? Italia. Psicoanálisis, 33(1), 77-88.

Montaño, L. (2006). La Cirugía Estética y las Modificaciones de la Imagen Corporal. Interpsiquis, intersalud, 7. Recuperado el 20 de Abril de http://www.psiquiatria. com/bibliopsiquis/handle/10401/3702

Moreno, M., \& Ortiz, G. (2009). Trastorno alimentario y su relación con la imagen corporal y la autoestima en adolescentes. Terapia Psicológica, 27(2), 181-190. Recuperado el 15 de Enero de 2014 de http://teps.cl/files/2010/05/art04.pdf

Otero, M., Fernández, M., \& Castro, Y. (2004). Influencia de la imagen corporal y la autoestima en la experiencia sexual de estudiantes universitarias sin trastornos alimentarios. International Journal of clinical and Health Psychology, 4(2), 357-370. 
Pérez, A. (2009). Cuerpos Tatuados, "Almas" Tatuadas: Nuevas Formas De Subjetividad En La Contemporaneidad. Colombia. Revista Colombiana de Antropología, 45(1), 64-69.

Pérez, V. \& Sánchez, R. (2001). Las Concepciones Del Cuerpo Y Su Influencia En El Currículum De La Educación Física. Revista Digital-Buenos Aires, 6 (33), 1-2.

Pierrat, J., Guillon, É., Joly, M., \& Lesven, D. (2000). Les Hommes Illustrés: Le Tatouage Des Origines À Nos Jours. Larivière. Recuperado de http:// www.decitre.fr/livres/les-hommesillustres-9782907051965.html

Pozgain, I., Barkic, J., Filakovic, P., \& Koic, O. (2004). Tattoo And Personality Traits In Croatian Veterans. Croatia. Yonsei Medical Journal, 45, 300-305.

Raich, R. (2004). Una Perspectiva Desde La Psicología De La Salud De La Imagen Corporal. Colombia. Avances en Psicología Latinoamericana, 22(1).15-27.

Rasines, C. (2012). Relación de la Cirugía Estética con los Niveles de Autoestima y Autoconcepto en Mujeres Adolescentes. Universidad Centro occidental, Online, Recuperado el 22 de Abril de http://bibhumartes.ucla. edu.ve/DB/bcucla/edocs/repositorio/ TEGBF7243S35R382012.pdf

Roberts, T., \& Ryan, S. (2002). Tattooing And High-Risk Behavior In adolescents. Pediatrics, 110(6), 1058-1063.

Rodríguez, R., Aguilar, A., Rodríguez, R., \& Rodríguez, L. M. (2012). Necrosis y absceso de piel secundario tatuaje permanente en un adulto joven. Medicina Interna de México, 28(3), 298.

Salinas, L. (1994). La Construcción Social Del Cuerpo. Revista Española de Investigaciones Sociológicas, 68, 85-96.

Sarwer, D., Wadden, T., Pertschuk, M., \& Whitaker, L. (1988). Body image dissatisfaction and body dysmorphic disorder in 100 cosmetic surgery patients. Plastic and Reconstructive Surgery, 101 (6), 1644-1649.

Sastre, A. (2011). Cuerpos Que Narran: la práctica del tatuaje y el proceso de subjetivación. Colombia. Revista diversitas - Perspectivas en Psicología, 7 (1), 179- 191.

Schilder, P., Krapf, E., Loedel, E., \& Corsico, R. (1983). Imagen y apariencia del cuerpo humano: estudios sobre las energías constructivas de la psique. Barcelona: Paidós.

Sepúlveda, A., Gandarillas, A., \& Carrobles, J. (2004). Prevalencia de trastornos del comportamiento alimentario en la población universitaria. Quinto congreso virtual de psiquiatría Online, recuperado el 20 de Abril de 2014 de http://www. psiquiatria.com/tr_personalidad_y_ habitos/alimentacion_trastornos_del prevalencia-de-trastornos-delcomportamiento-alimentario-en-lapoblacion-universitarial.

Soto, G., \& Morett, A. (2004). Tatuajes y perforaciones en adolescentes. ¿Símbolo de status o síntoma de alarma? Presentación de dos casos extremos. Acta Pediátrica de México, 25(3)184-190. 
Stephens, M. (2003). Behavioral risks associated with tattooing. U.S. family medicine-kansascity,35(1),52-54.

Tausk, F., \& Nousari, H. (2001).Stress and the skin. Archives of Dermatology, 137(1), 78-82.

Tesone, J. (2000). El Tatuaje y el Escudo de Perseo. Comisión Directiva, 23(2), 179-195.

Torres, V. (2007).Utilizando el cuerpo: una mirada antropológica del tatuaje. Uruguay. Taller de antropología social II. Recuperado el 26 de Septiembre de 2014 de http://www.modartteam.com/ Espanol/Proyectos_Eventos/Valentina_ Brena_Utilizando_el_Cuerpo_Una_ mirada_antropologica_del_Tatuaje.pdf
Vargas, L. (2011). Percepción de la imagen corporal en adolescentes comparación por género. Ciencia y cultura física: Un reto del presente. Online, Recuperado el 15 de Mayo de 2014 de http://promepca. sep.gob.mx/archivospdf/produccion/ Producto1350586.PDF

Yépez, R., \& Arzápalo, R. (2007). La práctica cultural de modificar el cuerpo como un texto de información e interpretación social para la antropología física: una perspectiva Semiótica. Papeles de trabajoCentro de Estudios Interdisciplinarios en Etnolingüísticay Antropología SocioCultural, (15), 75-108. 